Enrico G. Ferro, MD

Department of Medicine, Brigham

and Women's Hospital, Boston, MA;

Harvard Medical School, Boston, MA
Bertram Pitt, MD

University of Michigan, Ann Arbor, Ml; Emeritus

Professor of Medicine, University of Michigan

School of Medicine, Ann Arbor, Ml; Co-Chair,

SOLOIST trial
Deepak L. Bhatt, MD, MPH

Professor of Medicine, Harvard Medical School,

Boston, MA; Executive Director of Interventional

Cardiovascular Programs, Division of Cardiovascular

Medicine, Brigham and Women's Hospital, Boston,

MA; Chair, SOLOIST and SCORED trials

\title{
SGLT-2 inhibitors in heart failure: Time for broader eligibility and earlier initiation
}

I $\mathrm{n}$ recent years, the treatment of heart failure with reduced ejection fraction (HFrEF) has been revolutionized by collaborative efforts among healthcare practitioners, pharmaceutical industry leaders, and regulators regarding the use of sodium-glucose cotransporter 2 (SGLT-2) inhibitors. ${ }^{1}$ A series of cardiovascular outcome trials led to the discovery of powerful and broad cardiorenal benefits associated with this class of drugs, originally developed as noninsulin therapy for type 2 diabetes mellitus. Studies have shown that SGLT-2 inhibitors result in statistically significant reductions in the rates of major adverse cardiovascular events, major adverse kidney events, and hospitalizations for heart failure in patients with diabetes. ${ }^{2-6}$

Notably, these benefits seemed to apply to all patients with HFrEF, whether or not they had diabetes. This initial observation led to a second wave of cardiovascular outcome trials focused on patients with chronic HFrEF (ejection fraction $\leq 40 \%$ ) managed in ambulatory settings. In these trials, ${ }^{7,8}$ SGLT-2 inhibitors dapagliflozin and empagliflozin conferred impressive reductions in risks of cardiovascular mortality and hospitalizations for heart failure, translating to a number-needed-to-treat of about 20 patients per year, regardless of the presence or absence of diabetes.

\section{HOW DO SGLT-2 INHIBITORS WORK?}

The broad cardiorenal benefits of SGLT-2 inhibitors are mediated by several beneficial mechanisms in addition to the well-characterdoi:10.3949/ccjm.88a.21045 ized reduction in glucose reabsorption in the proximal tubule of the kidney, the pathway originally targeted for noninsulin treatment of hyperglycemia. ${ }^{9}, 10$ SGLT-2 is a cotransporter of both glucose and sodium; thus, its inhibition promotes diuresis and reduces preload, afterload, and blood pressure. ${ }^{11}$ It may also directly increase renal erythropoietin and the oxygencarrying capacity of the blood, perhaps mimicking benefits seen with intravenous iron in patients with HFrEF. ${ }^{12}$ In addition to the kidneys, constant glycosuria by itself has a direct cardiac benefit by shifting metabolism in favor of oxidation of free fatty acids, which in turn optimizes mitochondrial function in cardiac myocytes (improving contractile function) and reduces epicardial fat (decreasing noxious inflammation and fibrosis associated with heart failure). These mechanisms may explain the reduction in left ventricular mass index, a known predictor of major adverse cardiovascular events, seen on cardiac magnetic resonance imaging and associated with empagliflozin and dapagliflozin use. ${ }^{11,13}$ Furthermore, SGLT-2 inhibitors may cross-react with cardiac sodiumhydrogen exchangers, which has been linked to decreased arrhythmia burden. ${ }^{14}$

\section{CLINICAL ROLES ARE EXPANDING}

While the synergistic mechanisms of action of SGLT-2 inhibitors require further characterization, their safety and net clinical benefits have been so rigorously demonstrated that all major international guidelines now recommend them as treatment for diabetes and associated kidney disease, administered 


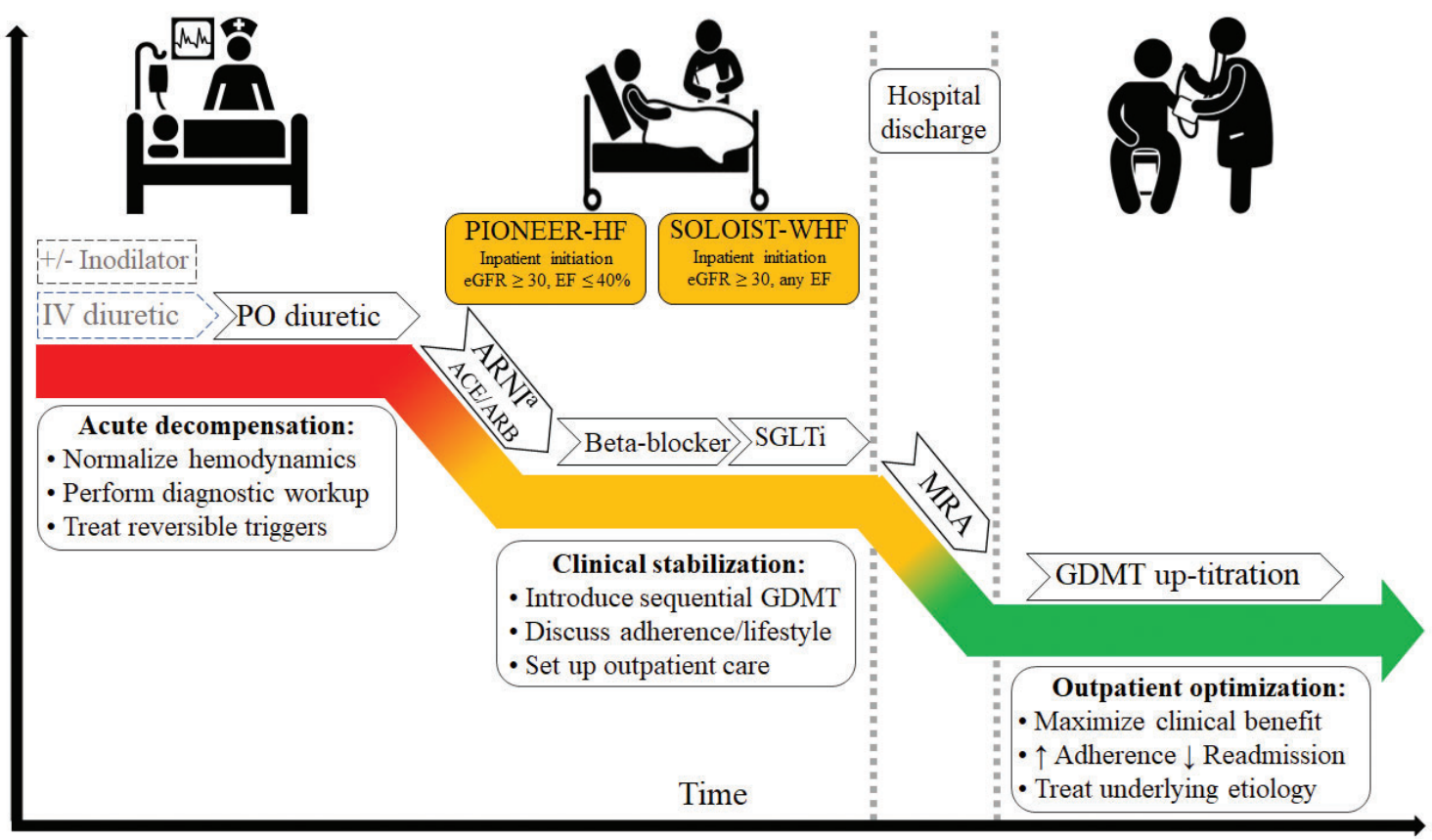

Figure 1. Guideline-directed medical therapy for a hospitalized patient with heart failure, showing early initiation of SGLT-2 inhibitor therapy. If an ARNI cannot be used as first-line therapy because it is contraindicated or not tolerated, consider an angiotensin-converting enzyme inhibitor or angiotensin receptor blocker.

$\mathrm{ACE}=$ angiotensin-converting enzyme inhibitor; $\mathrm{ARB}=$ angiotensin receptor blocker; $\mathrm{ARNI}=$ angiotensin receptor-neprilysin inhibitor; $\mathrm{EF}=$ ejection fraction; eGFR = estimated glomerular filtration rate $\left(\mathrm{mL} / \mathrm{min} / 1.73 \mathrm{~m}^{2}\right) ; \mathrm{GDMT}=$ guideline-directed medical therapy; IV = intravenous; MRA = mineralocorticoid receptor antagonist; PO = per os (by mouth); PIONEER-HF = Comparison of Sacubitril-Valsartan Versus Enalapril on Effect on NT-proBNP in Patients Stabilized From an Acute Heart Failure Episode; SGLTi = sodium-glucose cotransporter inhibitor; SOLOIST-WHF = Effect of Sotagliflozin on Cardiovascular Events in Patients With Type 2 Diabetes Post Worsening Heart Failure

concurrently with metformin or even as firstline therapy. ${ }^{15,16}$ Given the overwhelming evidence of benefit in all patients with HFrEF, whether or not they have diabetes, ${ }^{7,8}$ consensus statements from the cardiology community uniformly encourage physicians to prioritize SGLT-2 inhibitor initiation in this patient population, potentially even as the initial therapy alongside beta-blockers. ${ }^{17,18}$ In addition, the accumulating evidence of benefit in patients with chronic proteinuric kidney disease regardless of diabetes ${ }^{6,19,20}$ led the US Food and Drug Administration to approve dapagliflozin to treat patients with chronic kidney disease regardless of diabetes, making it the first SGLT-2 inhibitor to achieve a triple indication: type 2 diabetes, HFrEF, or chronic kidney disease. This will likely lead to guideline updates and significant increases in patient eligibility.

While other SGLT-2 inhibitors have not yet received such broad regulatory approval, the evidence generated by clinical trials to date suggests an overall class effect that applies to all available agents. ${ }^{21}$

\section{INCREASINGLY PROVEN, YET UNDERUSED}

Despite the impressive cardiorenal benefits of SGLT-2 inhibitors and endorsement by many medical societies, real-world use of these drugs is low. A retrospective analysis of 5,006 US patients with high cardiovascular risk examined SGLT-2 inhibitor use from 2016 to 2018,22 about 1 to 2 years after publication of the landmark SGLT-2 inhibitor trials, ${ }^{2,3}$ and found use of angiotensin-converting enzyme (ACE) inhibitors and angiotensin receptor blockers to be high ( $72 \%$ of patients), whereas concurrent use of SGLT-2 inhibitors was alarmingly low at $9 \% .{ }^{22}$ SGLT-2 inhibitors are particularly under- 
used outside of endocrine practices. A retrospective analysis of approximately $1,800 \mathrm{pa}$ tients who were started on SGLT-2 inhibitors in 2017 in Massachusetts found that 45.4\% of patients were started on this treatment by endocrinologists, $22.7 \%$ by primary care physicians, and only $4.5 \%$ by cardiologists. ${ }^{23}$

Targeted interventions are needed to increase SGLT-2 inhibitor use in patients with diabetes and nondiabetes proteinuric kidney disease, as trials have demonstrated substantial benefit in patients with increasingly lower estimated glomerular filtration rate and milder proteinuria, thus expanding eligibility. ${ }^{19,20,24,25}$

\section{THE FUTURE FOR SGLT-2 INHIBITORS IN HEART FAILURE THERAPY}

Patients hospitalized for HFrEF represent a population in whom an updated guideline-directed medical therapy protocol can be safely started or advanced. This protocol calls for early addition of SGLT-2 inhibitor therapy (Figure 1), a strategy shown to reduce morbidity and mortality as early as 30 days after initiation. ${ }^{18,26}$ Inhospital initiation of therapy with an SGLT-2 inhibitor is also an independent predictor of higher adherence to therapy in patients with worsening heart failure, ${ }^{27}$ thus maximizing the clinical benefit of these agents.

The conventional therapeutic approach was to start with an ACE inhibitor or angiotensin receptor blocker followed by a betablocker, mineralocorticoid receptor antagonist, and a neprilysin inhibitor, and then an SGLT-2 inhibitor, primarily in the outpatient setting. The feasibility and benefits of the conventional approach were based on established evidence from clinical trials. However, the evidence for SGLT-2 inhibitors from those trials was limited to patients with chronic ambulatory heart failure and excluded patients hospitalized with heart failure fewer than 4 weeks before enrollment. ${ }^{27}$

\section{SOLOIST-WHF trial: More evidence}

The clinical trial Effect of Sotagliflozin on Cardiovascular Events in Patients With Type 2 Diabetes Post Worsening Heart Failure (SOLOIST-WHF) ${ }^{28}$ enrolled 1,222 patients with diabetes and heart failure (reduced ejection fraction $[<50 \%]$ or preserved ejection fraction $[\geq 50 \%]$ ) with elevated $\mathrm{N}$-terminal B- type natriuretic peptide who were hospitalized for worsening heart failure and had been clinically stabilized, ie, no hypotension or need for supplemental oxygen, intravenous inotropic therapy, or intravenous diuretics. Patients were randomized to either sotagliflozin (an inhibitor of SGLT-1 and SGLT-2) or placebo.

The trial was terminated early due to loss of funding at the onset of the COVID-19 pandemic, resulting in a smaller sample size and shorter follow-up than anticipated. Nonetheless, after a median follow-up of 0.75 years, sotagliflozin recipients had a 33\% relative risk reduction $(P=.0009)$ and a $25 \%$ absolute risk reduction (translating to a number needed to treat of 4 patients for a year) in the primary end point of total cardiovascular deaths, hospitalizations for heart failure, and urgent visits for heart failure. ${ }^{28}$

Of note, the first dose of the trial medication was administered before discharge in about half of the patients and at a median of 2 days after discharge in the other half (with no major difference in safety issues compared with placebo). Overall, this trial demonstrated the feasibility, safety, and early clinical benefit of the in-hospital initiation of an SGLT-1 and SGLT-2 inhibitor, given that the cumulative incidence curves for the primary outcome were already significant by day 28 postrandomization. ${ }^{27,28}$

\section{WHAT'S THE CLINICAL IMPACT?}

We and others believe it is time for a shift in the timing and sequence of SGLT-2 inhibitor therapy. ${ }^{17,18,27}$ Impressive reductions in major adverse cardiovascular events, major adverse kidney events, and hospitalizations for heart failure consistently shown in SGLT-2 inhibitor trials, ${ }^{7,8,12}$ together with the safety and early benefit of in-hospital initiation shown by SOLOIST-WHF, ${ }^{28}$ provide rigorous evidence to support initiating SGLT-2 inhibitors as first-line treatment for $\mathrm{HFrEF}$ as soon as the patient is clinically stable. ${ }^{26}$

For example, as part of guideline-directed medical therapy, SGLT-2 inhibitor therapy can be prioritized as the first agent coupled with beta-blockers, which continue to be the single most effective drug class for $\mathrm{HFrEF} \cdot{ }^{18}$ As the other 2 cornerstones of HFrEF therapy (an

\section{It is time for a shift in the timing and sequence of SGLT-2 inhibitor therapy in patients with heart failure}


angiotensin receptor-neprilysin inhibitor and a mineralocorticoid receptor agonist) are then added to this background of SGLT-2 inhibition, the diuretic effect of SGLT-2 inhibition can further reduce the risk of hyperkalemia, increasing safety and tolerability. ${ }^{18}$ Others, including the American College of Cardiology, ${ }^{17}$ propose initiating renin-angiotensinaldosterone system inhibitors as the first step, prioritizing an angiotensin receptor-neprilysin inhibitor over an ACE inhibitor or angiotensin receptor blocker, as this may be better tolerated when the patient is still mildly congested and approaching clinical stabilization, followed by a beta-blocker and SGLT-2 inhibitor.

\section{HEART FAILURE WITH PRESERVED EJECTION FRACTION}

The SOLOIST-WHF trial ${ }^{28}$ also uncovered an additional benefit of SGLT inhibition that may further solidify the role of this drug class as the pillar of modern heart failure treatment-ie, therapeutic applications to patients with preserved ejection fraction.

The SOLOIST-WHF trial ${ }^{28}$ was the first

Over the past

5 years, SGLT-2 inhibitors have changed the treatment

paradigm for diabetes, chronic kidney disease, and heart failure heart failure-focused trial of SGLT inhibition to enroll patients with preserved ejection fraction, in order to investigate whether the beneficial effect of this drug class might apply irrespective of the patient's ejection fraction. Although the study planned to enroll $50 \%$ of patients with a preserved ejection fraction of $50 \%$ or greater, its early termination resulted in only $20 \%$ of the final sample size meeting this criterion. ${ }^{28}$ Despite the modest sample size, there was no evidence of heterogeneity of treatment effect according to ejection fraction. ${ }^{27,28}$ This promising finding suggested that SGLT-2 inhibitors may also become the first therapeutic option for heart failure with preserved ejection fraction, a very common yet elusive disease for which no treatment had yet convincingly reduced rates of morbidity or mortality.

Recently, the EMPEROR-Preserved trial ${ }^{29}$ showed a significant reduction in the rate of cardiovascular death or hospitalization for heart failure in patients with preserved ejection fraction, extending the findings of SOLOIST-WHF to include not only patients with diabetes but also those without diabetes. A trial of dapagliflozin in a similar population should report relatively soon. Therefore, it appears likely that SGLT-2 inhibitors as a class will now be a therapy for heart failure with either preserved or reduced ejection fraction in those with or without diabetes. ${ }^{29}$

\section{ADDITIONAL CARDIOVASCULAR BENEFITS}

Sotagliflozin inhibits the SGLT-1 receptor as well as the SGLT-2 receptor. The SGLT-1 transporter mediates only $10 \%$ of kidney glucose reabsorption. Its primary role is in the small intestines, where its inhibition delays glucose absorption and reduces postprandial glycemia. ${ }^{9}$ SGLT-1 inhibition, whether alone or together with SGLT-2 inhibition, may confer additional cardiovascular benefit. This observation is supported by the Effect of Sotagliflozin on Cardiovascular and Renal Events in Patients With Type 2 Diabetes and Moderate Renal Impairment Who Are at Cardiovascular Risk (SCORED) trial, ${ }^{24}$ in which sotagliflozin demonstrated an early and significant reduction in myocardial infarction and stroke, with a relative risk reduction (compared with placebo) that seemed larger compared with more selective SGLT-2 inhibitors, such as empagliflozin or canagliflozin, in similar patient populations. ${ }^{2,3,21,24}$ While promising, these findings warrant additional study to determine if SGLT-1 inhibition really does add to SGLT-2 inhibition in terms of cardiovascular risk reduction.

\section{STILL AN UNDERUSED RESOURCE}

Over the past 5 years, SGLT-2 inhibitors have changed the treatment paradigm for patients with diabetes, chronic kidney disease, and heart failure. These drugs have become a powerful resource that is shared by primary care, endocrinology, cardiology, and nephrology specialists, yet they remain vastly underused in clinical practice despite their broad cardiorenal benefits. By providing the evidence and rationale for use of SGLT-2 inhibitors in this patient population, we hope that practitioners from all specialties will readily integrate these agents into their routine clinical practice. 


\section{DISCLOSURES}

Dr. Pitt served as co-chair of SOLOIST (Effect of Sotagliflozin on Cardiovascular Events in Patients With Type 2 Diabetes Post Worsening Heart Failure) and was on the executive committee of SCORED (Effect of Sotagliflozin on Cardiovascular and Renal Events in Patients With Type 2 Diabetes and Moderate Renal ulting fees from Sanofi/Lexicon. In addition, Dr. Pitt discloses the following: consulting fees from Bayer, Astra Zeneca, Boehringer Ingelheim/Lilly, and Phasebio; and consulting fees and stock options from SCPharmaceuticals, SQinnovations, G3pharmaceuticals, Relypsa/Vifor, Cereno scientific, KBP Pharmaceuticals, Sarfez, Tricida, Proton Intel, and Brainstorm Medical. Dr. Pitt is chairman of the steering Committee for the National Heart Lung, and Blood Institute's TRANSFORM (Torsemide Comparison With Furosemide For Management Heart Failure) trial and co-chair of SPIRRIT (Spironolactone Initiation Registry Randomized Interventional Trial) from the National Heart, Lung, and Blood Institute-Swedish Heart Foundation. He holds US Patent No. 9931412 on site-specific delivery of eplerenone to the myocardium and has a pending US Patent $(63 / 045,784)$ on histone acetylation-modulating agents for the treatment and protection of organ damage.

Dr. Deepak L. Bhatt discloses the following relationships: Advisory Board: Cardax, CellProthera, Cereno Scientific, Elsevier Practice Update Cardiology, Janssen, Level Ex, Medscape Cardiology, MyoKardia, Novo Nordisk, PhaseBio PLx Pharma, Regado Biosciences; Board of Directors: Boston VA Research Institute, Society of Cardiovascular Patient Care, TobeSoft; Chair: American Heart Association Quality Oversight Committee; Data Monitoring Committees: Baim Institute for Clinical Research (formerly Harvard Clinical Research Institute for the PORTICO trial, funded by St. Jude Medical, nOw Abbott), Cleveland Clinic (including for the ExCEED trial, funded by Edwards), Contego Medical (Chair, PERFORMANCE 2), Duke Clinical Research Institute, Mayo Clinic, Mount Sinai School of Medicine (for the ENVISAGE trial, funded by Daiichi Sankyo),

\section{REFERENCES}

1. Ferro EG, Michos ED, Bhatt DL, Lincoff AM, Elshazly MB. New decade, new FDA guidance for diabetes drug development: lessons learned and future directions. J Am Coll Cardiol 2020; 76(21):2522-2526. doi:10.1016/j.jacc.2020.09.590

2. Zinman B, Wanner C, Lachin JM, et al. Empagliflozin, cardiovascular outcomes, and mortality in type 2 diabetes. N Engl J Med 2015; 373(22):2117-2128. doi:10.1056/NEJMoa1504720

3. Neal B, Perkovic V, Mahaffey KW, et al. Canagliflozin and cardiovascular and renal events in type 2 diabetes. N Engl J Med 2017; 377(7):644-657. doi:10.1056/NEJMoa1611925

4. Wiviott SD, Raz I, Bonaca MP, et al. Dapagliflozin and cardiovascular outcomes in type 2 diabetes. $\mathrm{N}$ Engl J Med 2019; 380(4):347-357. doi:10.1056/NEJMoa1812389

5. Cannon CP, Pratley R, Dagogo-Jack S, et al. Cardiovascular outcomes with ertugliflozin in type 2 diabetes. N Engl J Med 2020; 383(15):1425-1435. doi:10.1056/NEJMoa2004967

6. Perkovic V, Jardine MJ, Neal B, et al. Canagliflozin and renal outcomes in type 2 diabetes and nephropathy. N Engl J Med 2019; 380(24):2295-2306. doi:10.1056/NEJMoa1811744

7. McMurray JJV, Solomon SD, Inzucchi SE, et al. Dapagliflozin in patients with heart failure and reduced ejection fraction. N Engl J Med 2019; 381(21):1995-2008. doi:10.1056/NEJMoa1911303

8. Packer M, Anker SD, Butler J, et al. Cardiovascular and renal outcomes with empagliflozin in heart failure. N Engl J Med 2020; 383(15):1413-1424.

doi:10.1056/NEJMoa2022190

9. Zelniker TA, Braunwald E. Mechanisms of cardiorenal effects of sodium-glucose cotransporter 2 inhibitors: JACC state-of-the-art review. J Am Coll Cardiol 2020; 75(4):422-434. doi:10.1016/j.jacc.2019.11.031

10. Tsushima Y, Lansang MC, Makin V. The role of SGLT-2 inhibitors in managing type 2 diabetes. Cleve Clin J Med 2020; 88(1):47-58. doi:10.3949/ccjm.88a.20088

11. Verma S, Mazer CD, Yan AT, et al. Effect of empagliflozin on left ventricular mass in patients with type
Population Health Research Institute; Honoraria: American College of Cardiology (Senior Associate Editor, Clinical Trials and News, ACC.org; Vice-Chair, ACC Accreditation Committee), Baim Institute for Clinical Research (formerly Harvard Clinical Research Institute; RE-DUAL PCI clinical trial steering committee funded by Boehringer Ingelheim; AEGIS-II executive committee funded by CSL Behring), Belvoir Publications (Editor in Chief, Harvard Heart Letter), Canadian Medical and Surgical Knowledge Translation Research Group (clinical trial steering committees), Duke Clinical Research Institute (clinical trial cal trial steering committees), Duke Clinical Research Institute (clinical trial
steering committees, including for the PRONOUNCE trial, funded by Ferring Pharmaceuticals), HMP Global (Editor in Chief, Journal of Invasive Cardiology), Journal of the American College of Cardiology (Guest Editor; Associate Editor), K2P (Co-Chair, interdisciplinary curriculum), Level Ex, Medtelligence/ReachMD (CME steering committees), MJH Life Sciences, Population Health Research Institute (for the COMPASS operations committee publications committee steering committee and USA national co-leader, funded by Bayer), Slack Publications (Chief Medical Editor, Cardiology Today's Intervention), Society of Publications (Chief Medical Editor, Cardiology Today's Intervention), Society of
Cardiovascular Patient Care (Secretary/Treasurer), WebMD (CME steering committees); Other: Clinical Cardiology (Deputy Editor), NCDR-ACTION Registry Steering Committee (Chair), VA CART Research and Publications Committee (Chair); Research Funding: Abbott, Afimmune, Amarin, Amgen, AstraZeneca, Bayer, Boehringer Ingelheim, Bristol-Myers Squibb, Cardax, Chiesi, CSL Behring, Eisai, Ethicon, Ferring Pharmaceuticals, Forest Laboratories, Fractyl, HLS Therapeutics, Idorsia, Ironwood, Ischemix, Janssen, Lexicon, Lilly, Medtronic, MyoKardia, Novo Nordisk, Owkin, Pfizer, PhaseBio, PLx Pharma, Regeneron, Roche, Sanofi, Synaptic, The Medicines Company; Royalties: Elsevier (Editor, Cardiovascular Intervention: A Companion to Braunwald's Heart Disease); Site Co-Investigator: Abbott, Biotronik, Boston Scientific, CSI, St. Jude Medical (now Abbott), Svelte; Trustee: American College of Cardiology; Unfunded Research: FlowCo, Merck, Takeda.

Dr. Ferro reports no relevant financial relationships which, in the context of his contributions, could be perceived as a potential conflict of interest.

2 diabetes mellitus and coronary artery disease: the EMPA-HEART CardioLink-6 randomized clinical trial. Circulation 2019; 140(21):1693-1702. doi:10.1161/CIRCULATIONAHA.119.042375

12. Bhatt DL, Verma S, Braunwald E. The DAPA-HF trial: a momentous victory in the war against heart failure. Cell Metab 2019; 30(5):847-849.

doi:10.1016/j.cmet.2019.10.008

13. Brown AJM, Gandy S, McCrimmon R, Houston JG, Struthers AD, Lang CC. A randomized controlled trial of dapagliflozin on left ventricular hypertrophy in people with type two diabetes: the DAPA-LVH trial. Eur Heart J 2020; 41(36):3421-3432. doi:10.1093/eurheartj/ehaa419

14. Fernandes GC, Fernandes A, Cardoso R, et al. Association of SGLT-2 inhibitors with arrhythmias and sudden cardiac death in patients with type 2 diabetes or heart failure: a meta-analysis of 34 randomized controlled trials. Heart Rhythm 2021; 18(7):1098-1105. doi:10.1016/j.hrthm.2021.03.028

15. Das SR, Everett BM, Birtcher KK, et al. 2020 Expert consensus decision pathway on novel therapies for cardiovascular risk reduction in patients with type 2 diabetes: a report of the American College of Cardiology Solution Set Oversight Committee. J Am Coll Cardiol 2020; 76(9):1117-1145. doi:10.1016/j.jacc.2020.05.037

16. Cosentino F, Grant PJ, Aboyans V, et al. 2019 ESC guidelines on diabetes, pre-diabetes, and cardiovascular diseases developed in collaboration with the EASD. Eur Heart J 2020; 41(2):255-323. doi:10.1093/eurheartj/ehz486

17. Writing Committee; Maddox TM, Januzzi JL Jr, Allen LA, et al. 2021 Update to the 2017 ACC expert consensus decision pathway for optimization of heart failure treatment: answers to 10 pivotal issues about heart failure with reduced ejection fraction: a report of the American College of Cardiology Solution Set Oversight Committee. J Am Coll Cardiol 2021; 77(6):772-810. doi:10.1016/j.jacc.2020.11.022

18. McMurray JJV, Packer M. How should we sequence the treatments for heart failure and a reduced ejection fraction?: a redefinition of evidence-based medicine. Circulation 2021; 143(9):875-877. doi:10.1161/CIRCULATIONAHA.120.052926 
19. Heerspink HJL, Stefánsson BV, Correa-Rotter R, et al. Dapagliflozin in patients with chronic kidney disease. N Engl J Med 2020; 383(15):1436-1446. doi:10.1056/NEJMoa2024816

20. Taliercio JJ, Thomas G, Nakhoul GN, Vachharajani TJ, Mehdi A. SGLT-2 inhibitors: a new era in managing diabetic kidney disease starts now. Cleve Clin J Med 2020; 88(1):59-63. doi:10.3949/ccjm.88a.20190

21. Kluger AY, Tecson KM, Barbin CM, et al. Cardiorenal outcomes in the CANVAS, DECLARE-TIMI 58, and EMPA-REG OUTCOME trials: a systematic review. Rev Cardiovasc Med 2018; 19(2):41-49. doi:10.31083/j.rcm.2018.02.907

22. Arnold SV, de Lemos JA, Rosenson RS, et al. Use of guideline-recommended risk reduction strategies among patients with diabetes and atherosclerotic cardiovascular disease. Circulation 2019; 140(7):618-620. doi:10.1161/CIRCULATIONAHA.119.041730

23. Vaduganathan M, Sathiyakumar V, Singh A, et al. Prescriber patterns of SGLT-2i after expansions of US Food and Drug Administration labeling. J Am Coll Cardiol 2018; 72(25):3370-3372. doi:10.1016/j.jacc.2018.08.2202

24. Bhatt DL, Szarek M, Pitt B, et al. Sotagliflozin in patients with diabetes and chronic kidney disease. N Engl J Med 2021; 384(2):129-139. doi:10.1056/NEJMoa2030186

25. Aggarwal R, Lu K, Chiu N, Bakris GL, Bhatt DL. US prevalence of individuals with diabetes and chronic kidney disease indicated for SGLT-2 inhibitor therapy. J Am Coll Cardiol 2020; 76(24):2907-2910.

doi:10.1016/j.jacc.2020.09.616

26. Vaduganathan M, Claggett BL, Jhund PS, et al. Estimating lifetime benefits of comprehensive disease-modifying pharmacological therapies in patients with heart failure with reduced ejection fraction: a comparative analysis of three randomised controlled trials. Lancet 2020; 396(10244):121-128. doi:10.1016/S0140-6736(20)30748-0

27. Verma S, Anker SD, Butler J, Bhatt DL. Early initiation of SGLT-2 inhibitors is important, irrespective of ejection fraction: SOLOIST-WHF in perspective. ESC Heart Fail 2020; 7(6):3261-3267. doi:10.1002/ehf2.13148

28. Bhatt DL, Szarek M, Steg PG, et al. Sotagliflozin in patients with diabetes and recent worsening heart failure. N Engl J Med 2021; 384(2):117-128. doi:10.1056/NEJMoa2030183.

29. Anker SD, Butler J, Filippatos G, et al. Empagliflozin in heart failure with a preserved ejection fraction. $\mathrm{N}$ Engl J Med 2021; 385(16):1451-1461. doi:10.1056/NEJMoa2107038.

Address: Deepak L. Bhatt, MD, MPH, Professor of Medicine, Harvard Medical School, 75 Francis Street, Boston, MA 02115; DLBhattMD@post.Harvard.edu 